\title{
Explaining Deep Learning Models Through Rule-Based Approximation and Visualization
}

\author{
Eduardo Soares, Plamen Angelov, Fellow, IEEE, Bruno Costa, Senior Member, IEEE, Marcos P Gerardo \\ Castro, Subramanya Nageshrao, Dimitar Filev, Fellow, IEEE
}

\begin{abstract}
This paper describes a novel approach to the problem of developing explainable machine learning models. We consider a Deep Reinforcement Learning (DRL) model representing a highway path planning policy for autonomous highway driving [1]. The model constitutes a mapping from the continuous multidimensional state space characterizing vehicle positions and velocities to a discrete set of actions in longitudinal and lateral direction. It is obtained by applying a customized version of the Double Deep Q-Network (DDQN) learning algorithm [2]. The main idea is to approximate the DRL model with a set of $I F \ldots T H E N$ rules that provide an alternative interpretable model, which is further enhanced by visualizing the rules. This concept is rationalized by the universal approximation properties of the rule-based models with fuzzy predicates. The proposed approach includes a learning engine composed of 0 -order fuzzy rules, which generalize locally around the prototypes by using multivariate function models. The adjacent (in the data space) prototypes, which correspond to the same action are further grouped and merged into so-called "MegaClouds" reducing significantly the number of fuzzy rules. The input selection method is based on ranking the density of the individual inputs. Experimental results show that the specific DRL agent can be interpreted by approximating with families of rules of different granularity. The method is computationally efficient and can be potentially extended to addressing the explainability of the broader set of fully connected deep neural network models.
\end{abstract}

Index Terms-Deep Reinforcement Learning, explainable AI, rule-based models, prototype- and density-based models, density-based input selection, autonomous driving.

\section{INTRODUCTION}

$\mathbf{R}$ ESEARCH on self-driving vehicles have made significant progress in recent years. However, a challenging topic in the field of self-driving cars concerns the transparency of the trained machine learning models which is needed for validation, verification, and certification [3]-[8]. The demand of understandable models involves interpretability and explainability of a trained agent in order to fully understand the knowledge encoded in them.

Linguistic IF ... THEN fuzzy rule-based models offer transparent insights in contrast to neural networks rather 'black box' approaches [9], [10]. Although these 'black box'

* Corresponding author

E. Soares* and P. Angelov are with LIRA, School of Computing and Communications, Lancaster University, Lancaster, LA1 4WA, UK, e.almeidasoares@lancaster.ac.uk, p.angelov@lancaster.ac.uk.

M. Castro, S. Nageshrao and D. Filev are with Ford Research and Innovation Center, Ford Motor Company, Palo Alto, CA 94304, USA, dfilev@ford.com,mgerard8@ford.com, snageshr@ford.com.

B. Costa, No Affiliation, brunuxcosta@gmail.com.

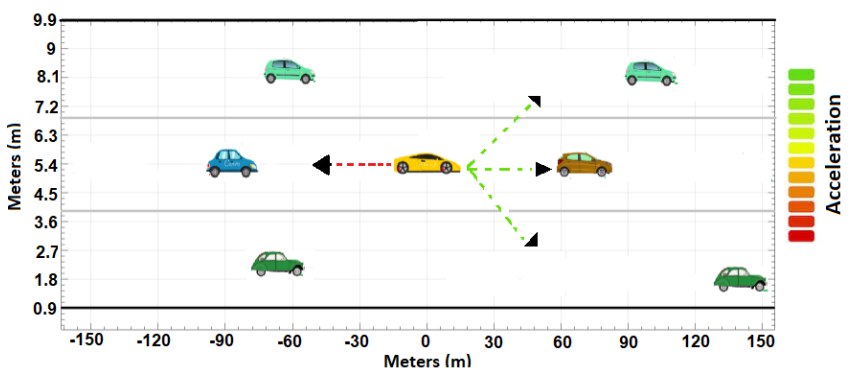

Fig. 1. Example of host (ego) and surrounding vehicles on a highway, where the host vehicle is represented by the center vehicle (yellow car). The forwards arrows indicate the possible directions which the ego vehicle can move. The backwards arrow indicates the brake maneuver.

models reach impressive classification and approximation accuracy, their nested non-linear structure makes them highly non-transparent [11], [12].

This paper proposes a new explainable self-organizing approach to transform a trained deep neural network model into a set of $I F \ldots$ THEN rules. We use a Deep Reinforcement Learning (DRL) model of the path planning policy for highway self-driving [1] to simulate data corresponding to driving scenarios. The model maps the set of continuous state variables characterizing the position and velocities of the ego vehicle (EV) and the surrounding vehicles on a divided highway into a set of discrete actions in longitudinal and lateral direction.

State variables include meaningful affordance indicators of the road situation such as the longitudinal and lateral position and velocity of the host vehicle and relative longitudinal and lateral positions and velocities of the surrounding vehicles. The output of the model is a set of eight possible decisions/actions in longitudinal (maintain, accelerate, brake, and hard brake) and lateral (lane keep, change lane to right, and change lane to left) directions - Fig. (1).

The main idea of this paper is to provide an approximation of the DRL model with an alternative interpretable model with a similar performance. Our approach is based on the following main concepts: i) the universal approximation ability of the rule-based models with fuzzy predicates; ii) the better interpretability of the prototype-based fuzzy rules (including visualization). The method allows to potentially learn the rules incrementally during the DRL training process.

The proposed method for learning rules expands from the previously published work [13], [14] by introducing a 
density-based method for selecting the most important inputs and a two-stage hierarchical approach to group the adjacent prototypes in the data space that correspond to the same action. These two novel techniques allow us to reduce the number of prototypes needed and improve the explainability. This is achieved both linguistically as a set of hierarchical $I F .$. THEN rules and through visualisation. In addition, we also propose a sequence of pair-wise decision process, rather than one decision for the recommended action, and a method for balancing the training data set to have approximately the same data samples per action. In combination, these innovations allow us to get an explainable approximation of the DRL agent decisions under multiple driving conditions and to summarize its performance in diverse situations.

In order to validate our concept, experiments were conducted using the DRL model provided by Ford Motor Company, see [1] for details. Results demonstrate that the proposed approach can achieve a computationally efficient, compact and easily explainable approximation of DRL models.

The focus of the paper is on the methodology for modeling of the multidimensional data set that is obtained through approximation of the simulated DRL model. The method is not constrained to DRL models and can be extended and adapted to other type of deep learning structures and architectures as well as to learning from data generated by human drivers.

The remainder of this paper is structured as follows. Section II introduces the proposed method. The data employed in the analysis is presented in Section III. The results and the discussion are provided in Section IV. Section V concludes the paper.

\section{The Proposed ApProACH}

In this section, we will introduce the approach briefly describing the general architecture, learning and validation. Let $T=\left\{\left(\boldsymbol{x}_{k}, \boldsymbol{a}_{k}\right)\right\}_{k=1}^{N}$ be training data set with $\boldsymbol{x}_{k} \in \mathbb{R}^{n}$ denoting the state vector and $\boldsymbol{a}_{k} \in\{1, \ldots, A\}$ denoting the action vector for each $k \in\{1, \ldots, N\}$. The layered architecture (Fig. (2)) can be seen as a mapping, $f: \mathbb{R}^{n} \rightarrow \mathbb{R}^{A}$; $n$ is the number of inputs; $A$ is the number of actions; $i$ is the specific data sample/point $k ; N$ is the number of training data samples. Separate learning cycles are introduced for each action. Therefore, the data set is split into A sub-sets.

The learning process starts with analyzing the mutual proximity of the data [15]. As a result, a small number of prototypes are being selected which are actual data samples that are most representative locally. When prototypes are being formed only data samples that correspond to the same action are being considered. When prototypes that correspond to different actions are being put together in the data space a further level of analysis is being made, namely merging adjacent (in the data space) prototypes that correspond to the same action together forming so-called "MegaClouds". Finally, the MegaClouds can be visualized and also represented by IF...THEN rules. The general architecture of the proposed approach is given in Fig. (2).
As a result, we compose $A$ parallel $I F$...THEN rules, each of which corresponds to one of the $A$ actions and has the following form:

$$
\begin{array}{r}
\mathrm{R}_{l}: \text { IF }\left(\boldsymbol{x} \sim \boldsymbol{p}_{l}^{1}\right) \text { OR }\left(\boldsymbol{x} \sim \boldsymbol{p}_{l}^{2}\right) \text { OR } \ldots \text { OR }\left(\boldsymbol{x} \sim \boldsymbol{p}_{l}^{P_{l}}\right) \\
\text { THEN }(\text { action } l)
\end{array}
$$

where $\boldsymbol{p}_{l}^{j}\left(j \in\left\{1, \ldots, P_{l}\right\}\right)$ is the $j^{\text {th }}$ prototype of the $l^{\text {th }}$ action; $P_{l}$ is the number of identified prototypes that represent the $l^{\text {th }}$ action.

The identified prototypes are connected with logical "OR" (implemented as a $t$-conorm). Strictly speaking, each of the conditions within the IF...THEN rules are fuzzy rules on their own but all of them have the same consequent pointing to the same action. The so-called "winner takes all" principle is used to decide the action of the IF...THEN rule during the validation process.

In summary, the proposed method can be represented as a hierarchy (see Fig. (3)) where the bottom layer is the data set and the next layer up is composed of all the identified prototypes during the learning process, while the top layer of the structure consists of a much smaller sub-set of highly informative prototypes corresponding to MegaClouds.

In the following two subsections, the main steps of learning and validation are described.

\section{A. Learning rules from the data}

The proposed method learns the prototypes associated with each action in a separate loop. Therefore, the data set is split during the training into sub-sets.

As each IF...THEN rule is identified separately for each action, unless specifically declared otherwise, all the mathematical notations in the algorithm consider the $l^{\text {th }}$ action by default and the index $l$ is omitted for clarity.

Step 1. Standardize the newly observed data sample, $\boldsymbol{x}$. Standardization is performed on a per input basis:

$$
\widehat{x}(i)=\frac{x(i)-\mu(x(i))}{\sigma(x(i))}
$$

where $\widehat{x}(i)$ denotes the standardized value of the $i$-th input of the data sample; $\mu(x(i))$ denotes the mean of the $i$-th input and $\sigma(x(i))$ denotes the standard deviation of the $i$-th input; $\boldsymbol{\mu} \in \mathbb{R}^{n}$ denotes the vector of the mean values and $\boldsymbol{\sigma} \in \mathbb{R}^{n}$ denotes the vector of the standard deviations.

Following the standardization the data is being normalized converting it to the range $[0,1]$. Unity-based normalization of the $i$-th input is given by [16]:

$$
\bar{x}(i)=\frac{\widehat{x}(i)-\min (\widehat{x}(i))}{\max (\widehat{x}(i))-\min (\widehat{x}(i))}
$$

where $\bar{x}(i)$ denotes the normalized values of the $i$-th input.

Step 2. Initialize the algorithm meta-parameters with the first data sample, $\bar{x}_{1}$ observed: 


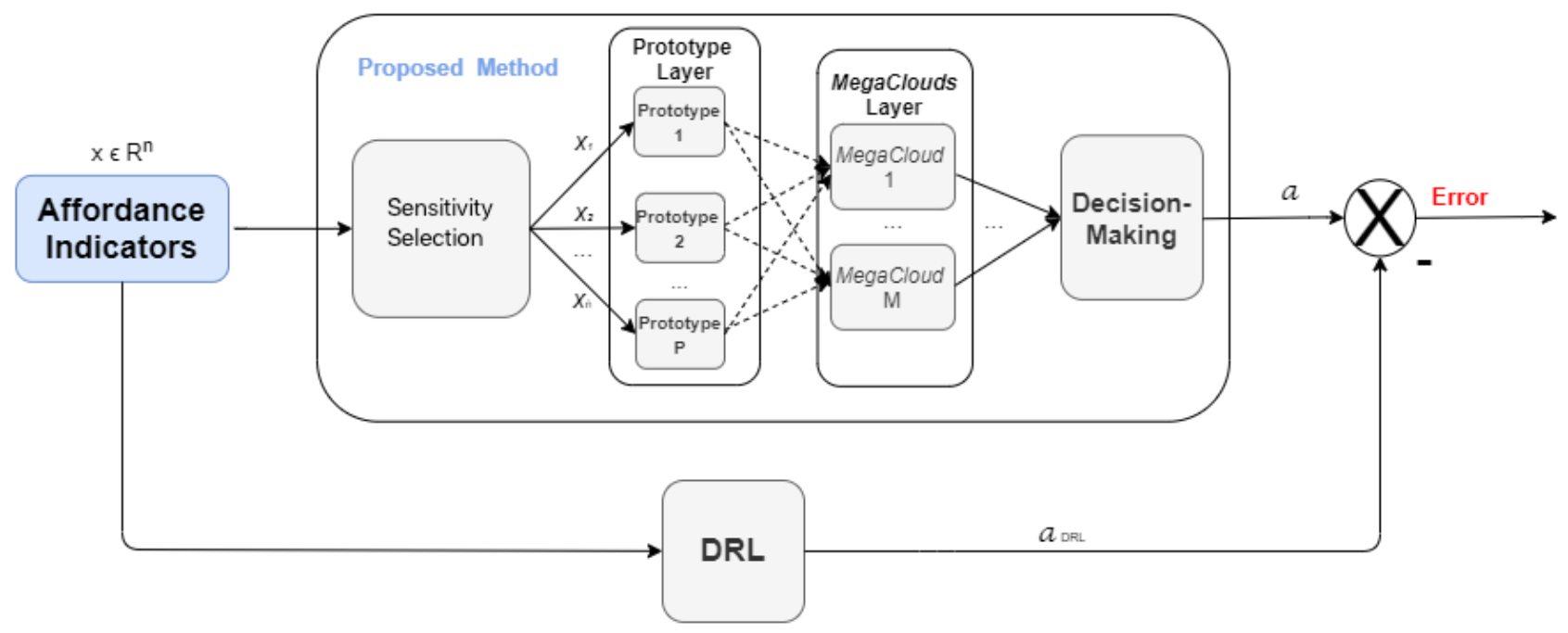

Fig. 2. General structure of the proposed approach. $a_{D R L}$ refers to the DRL output. The comparison between $a$ and $a_{D L R}$ is used to determine the accuracy of the proposed method.

$$
\begin{aligned}
& \boldsymbol{\mu}_{\mathbf{1}} \leftarrow \overline{\boldsymbol{x}}_{1} ; \quad P \leftarrow 1 ; \quad \boldsymbol{p}^{1} \leftarrow \overline{\boldsymbol{x}}_{1} \\
& \mathbf{C}^{1} \leftarrow\left\{\bar{x}_{1}\right\} ; \quad S^{1} \leftarrow 1 ; \quad r^{1} \leftarrow r^{o} ;
\end{aligned}
$$

These include: i) the mean being initialized with the first normalized data point; ii) the number of prototypes being set to 1 ; iii) the first prototype being initialized with the first data point; iv) initialize the first so-called data cloud, $\mathrm{C}^{1}$ as a set of data points that are associated with the first prototype (data clouds were defined in [17] as set of data points described by a prototype and differ somewhat form clusters by shape and boundaries and other properties); v) the so-called support of the data cloud $S^{1}$ defined as the number of data points associated with a certain data cloud [15]; vi) the radius of the area of influence around the prototype, $r^{1}$, in this paper we initialize it with $r^{o}=0.5$. In a multidimensional space $r^{O}=0.5$ is reasonable (not too low to avoid getting significant number of prototypes and not too high to allow a certain level of detail and granularity). Notice that $r^{1}$ is the only meta-parameter, its value is automatically determined by the algorithm. However, it is not user-defined and problemspecific as it only refers to an initial value which later will be updated with the real data.

Based on this initialization define the first IF...THEN rule for the given ( $l$-th action) as follows:

$$
\left.\mathbf{R}_{\boldsymbol{l}}: \quad \text { IF }\left(\boldsymbol{x} \sim \boldsymbol{p}_{l}^{1}\right) \text { THEN (action } l\right)
$$

Step 3. Calculate the data density at the current data point, $\overline{\boldsymbol{x}}_{k} ; k \in\{1, \ldots, N\}$. Starting from the mutual distances (Euclidean or Mahalanobis type) between the data points (samples) in the feature space it can be demonstrated theoretically [9] that the data density takes the form of a Cauchy type function as in Eq. (6).

$$
D\left(\bar{x}_{k}\right)=\frac{1}{1+\frac{\left\|\bar{x}_{k}-\mu_{N}\right\|^{2}}{\left\|\sigma_{N}\right\|^{2}}} ;
$$

where $D$ is the data density, and $\sigma_{N}$ denotes the standard deviation.

In this step we also identify the prototype $\boldsymbol{p}^{j^{*}}$ that is nearest to $\overline{\boldsymbol{x}}_{k}$ :

$$
j^{*}=\underset{j \in\{1, \ldots, P\}}{\operatorname{argmin}}\left\{\left\|\overline{\boldsymbol{x}}_{k}-\boldsymbol{p}^{j}\right\|^{2}\right\}
$$

Then, using the density and the distance to the nearest prototype, $p^{j}$, we check the following condition [9] based on which we determine if the current data point is going to be added to the set of prototypes or not. If the condition is met, go to Step 4; otherwise, go to Step 5.

$$
\begin{array}{r}
\text { IF }\left(D\left(\overline{\boldsymbol{x}}_{k}\right) \geq \max _{j \in\{1, \ldots, P\}}\left(D\left(\boldsymbol{p}^{j}\right)\right)\right) \\
\text { OR }\left(D\left(\overline{\boldsymbol{x}}_{k}\right) \leq \min _{j \in\{1, \ldots, P\}}\left(D\left(\boldsymbol{p}^{j}\right)\right)\right) \\
\text { OR }\left(\left\|\boldsymbol{p}^{j^{*}}-\overline{\boldsymbol{x}}_{k}\right\|>r^{j^{*}}\right) \\
\text { THEN (add a new data cloud) }
\end{array}
$$

where $D\left(p^{j}\right)$ is density of the nearest prototype, $p^{j}$.

Step 4. Add a new data cloud:

$$
\begin{array}{r}
P \leftarrow P+1 ; \quad \mathbf{C}^{P} \leftarrow\left\{\overline{\boldsymbol{x}}_{k}\right\} ; \\
\boldsymbol{p}^{P} \leftarrow \bar{x}_{k} ; \quad S^{P} \leftarrow 1 ; r^{P} \leftarrow r^{o} ;
\end{array}
$$

Then, go to Step 6.

Step 5. Update the meta-parameters of the nearest data cloud:

$$
\begin{array}{r}
C^{j^{*}} \leftarrow C^{j^{*}}+\left\{\overline{\boldsymbol{x}}_{\boldsymbol{k}}\right\} \\
\boldsymbol{p}^{j^{*}} \leftarrow \frac{S^{j^{*}}}{S^{j^{*}}+1} \boldsymbol{p}^{j^{*}}+\frac{S^{j^{*}}}{S^{j^{*}}+1} \overline{\boldsymbol{x}}_{k} \\
S^{j^{*}} \leftarrow S^{j^{*}}+1 \\
r^{j^{*}} \leftarrow \sqrt{\frac{\left(r^{j^{*}}\right)^{2}+\left\|\sigma^{j^{*}}\right\|}{2}} ;
\end{array}
$$


The radius $r^{j^{*}}$ of the area of influence $C^{j^{*}}$ is recursively updated to allow data clouds to converge to the local areas where data samples are densely distributed. Prototypes $p^{j^{*}}$ are also updated through Eq. (6).

Step 6. Update the IF...THEN rule, $\mathbf{R}_{l}$ with the identified prototypes:

$$
\begin{array}{r}
\mathbf{R}_{l}: \quad \text { IF }\left(\boldsymbol{x} \sim \boldsymbol{p}_{l}^{1}\right) \quad \text { OR }\left(\boldsymbol{x} \sim \boldsymbol{p}_{l}^{2}\right) \quad \text { OR } \ldots \text { OR }\left(\boldsymbol{x} \sim \boldsymbol{p}_{l}^{P}\right) \\
\text { THEN }(\text { action l) }
\end{array}
$$

\section{B. Hierarchical organisation of the prototypes}

Prototypes are organized in a hierarchical manner (Fig. (3)). At the bottom layer of the hierarchical architecture is the raw data set. In the layer above it is the set of prototypes (which are selected data points/samples). Each of the prototypes is a focal point in the data space forming data clouds, see Fig. (4) shaping the so-called Voronoi tessellation [18]. Since the prototypes are defined in isolation (per action) once they are put together in the data space quite often data clouds that correspond to the same action (describing IF...THEN rules with the same consequent) are adjacent. This allows us to merge these data clouds and, respectively fuzzy IF...THEN rules into so-called "MegaClouds" which have the same consequent (THEN part) and, respectively correspond to the same action. In this way, we minimise the amount of $I F \ldots$ THEN rules and improve the interpretablity of the model. We illustrate this in Fig. (3).

The membership function (MF) to a MegaCloud is formed as a Minkowski type kernel [19]. $\lambda$ is the parameter of the kernel such that $\lambda \in(-\infty, \infty)$. We found experimentally that order of $\lambda=6$ gives best results in terms of accuracy. This can be explained with the fact that the higher the order of $\lambda$ the more narrow the local Cauchy-type function becomes and less its generalization. However, the lower the order of $\lambda$ is the less accurate it is as it starts to blur with the neighbouring functions that are centred at other prototypes:

$$
M F^{k}\left(\overline{\boldsymbol{x}}_{i}\right)=\left(\frac{1}{P^{k}} \sum_{j=1}^{P^{k}}\left(\frac{1}{1+\frac{\left\|\overline{\boldsymbol{x}}_{i}-\boldsymbol{p}^{j}\right\|^{2}}{\left\|\boldsymbol{\sigma}^{2}\right\|}}\right)^{\lambda}\right)^{\frac{1}{\lambda}}
$$

where $M F^{k}$ is the degree of membership of the data point, $\bar{x}_{i}$ to the $k$-th MegaCloud. The membership function is multimodal: each of its peaks is located at one of the prototypes, defined by a Cauchy-type function as described in Eq. (6) One can see that the prototypes, identified earlier, $\boldsymbol{p}$ are the parameters of the MF. One can, however, find new parameters of the MegaClouds - the most obvious choice is the mean of each MegaCloud (since these Voronoi tessellation areas are adjacent by definition they form a larger area, see Fig. (4)), where $\boldsymbol{m}^{M}$ is the mean, $\sum_{j=1}^{P} \frac{p^{j}}{P}$, of the $M$-th MegaCloud associated with the $l^{t h}$ action; $\boldsymbol{m} \in \mathbb{R}^{n}$.

The IF...THEN rules over the MegaClouds have the same form, but the key difference is that the number of conditions linked with T-conorm/disjunction (logical OR) are much less. They have the following format:

$$
\begin{array}{r}
\mathbf{R}_{l}: \quad \text { IF }\left(\boldsymbol{x} \sim \boldsymbol{m}_{l}^{1}\right) \quad \text { OR } \ldots \text { OR }\left(\boldsymbol{x} \sim \boldsymbol{m}_{l}^{M}\right) \\
\text { THEN }(\text { action } l)
\end{array}
$$

where $\boldsymbol{m}^{M}$ is the mean, $\sum_{j=1}^{P} \frac{p^{j}}{P}$, of the $M$-th MegaCloud associated with the $l^{t h}$ action; $\boldsymbol{m} \in \mathbb{R}^{n}$.

\section{Density-Based Input Selection}

Inputs ranking and selection is a technique to reduce the dimensionality of a problem. A subset of relevant or more descriptive inputs facilitates model interpretation, and can produce better results due to the elimination of inputs that may confound the uncovering of patterns, trends, and relationships.

In this paper we estimate the contribution of each input using the density of data per input:

$$
D(\bar{x}(i))=\frac{1}{1+\frac{\|\bar{x}(i)-\mu(i)\|^{2}}{(\sigma(i))^{2}}}
$$

where $D\left(\bar{x}_{(i)}\right)$ denotes the density for $i$-th input of $\bar{x}_{(i)} ;(i \in$ $\{1, \ldots, n\})$.

The cumulative effect across all data samples for each input can be obtained according to the Eq. (15).

$$
\Lambda(i)=\Sigma_{j=1}^{N} D\left(\bar{x}_{j}(i)\right) .
$$

The cumulative contribution for each input $\Lambda(i)$ can be ranked. The higher the value of $\Lambda(i)$ is for a particular input, the more descriptive and important is the $i$-th input [20]. The idea is that interesting inputs have higher density than other inputs - meaning that it conveys unique, different clean information, and as consequence it contributes more to the rule-based model result because the overlap between data clouds of different actions is less pronounced in these inputs. Less descriptive inputs are left one by one based on its $\Lambda(i)$ score until reduction in accuracy performance is noted. This sensitivity selection is helpful to reduce computational complexity which is an advantage especially in online implementation.

\section{Simulation DATA}

The data set used for learning the rule-based approximation was generated by simulating the DRL model described in [1]. It contains 256960 instances described by 20 different inputs as described by Table I. The data set is divided into 8 different actions, each action represent a different state of the ego vehicle. The description of the actions are given below:

- Action 1 (Maintain): 217494 samples

- Action 2 (Accelerate by $+2 \mathrm{~m} / \mathrm{s}^{2}$ ): 12706 samples

- Action 3 (Brake by $-2 \mathrm{~m} / \mathrm{s}^{2}$ ): 6033 samples

- Action 4 (Hard brake by $-4 m / s^{2}$ ): 4530 samples

- Action 5 (Lane change to left): 8078 samples 
MegaClouds layer (highly abstract prototypes):

Bottom Layer:

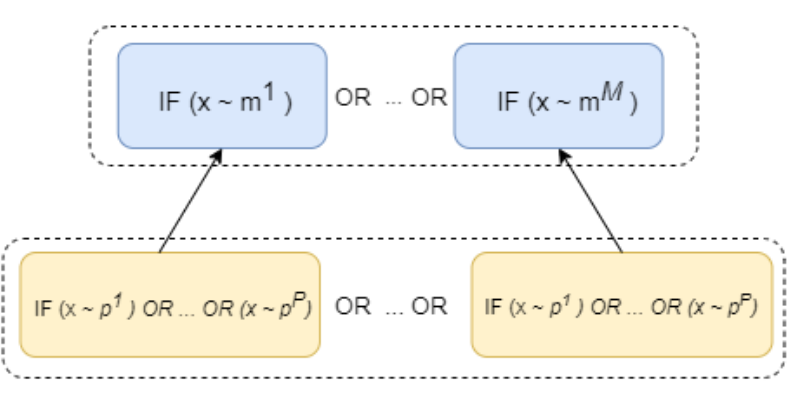

Fig. 3. Hierarchical structure - MegaClouds, where $\boldsymbol{m}^{M}$ is the mean of the $M$-th MegaCloud associated with the $l^{t h}$ action

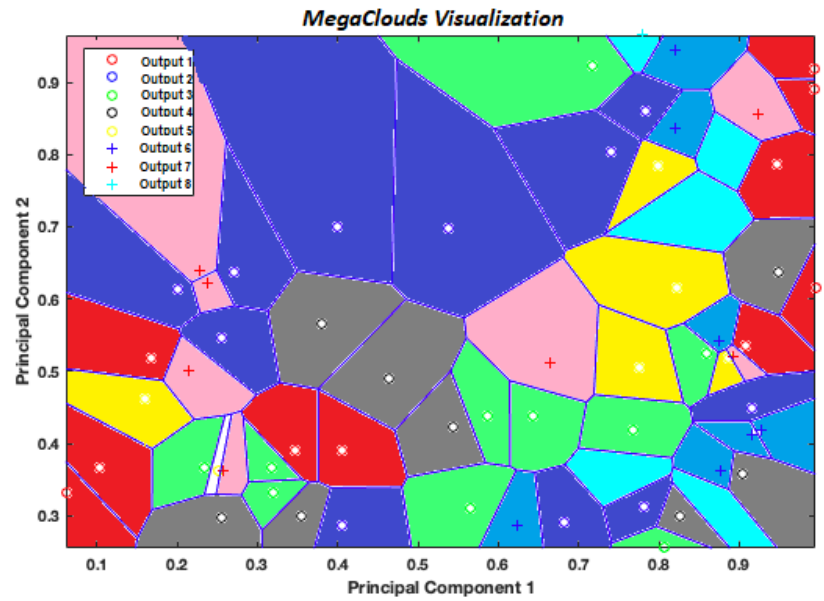

Fig. 4. MegaClouds visualization in terms of Voronoi Tesselation

- Action 6 (Lane change left and also brake by $-2 \mathrm{~m} / \mathrm{s}^{2}$ ): 213 samples

- Action 7 (Lane change right): 7704 samples

- Action 8 (Lane change right and also brake by $-2 \mathrm{~m} / \mathrm{s}^{2}$ ): 102 samples

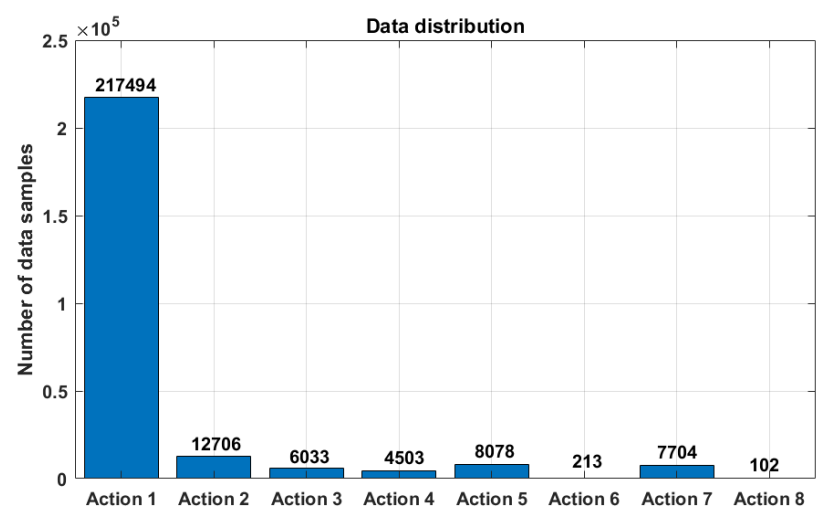

Fig. 5. Data Distribution in terms of different maneuvers/actions by the ego vehicle, showing the clearly data imbalance nature of the the data set.

The data set provided by Ford Motor Co. was obtained by a simulating DRL model representing driving policy of a
TABLE I

DESCRIPTION OF THE INPUTS

\begin{tabular}{c|c}
\hline Inputs & Description \\
\hline 1 & Ego lateral position \\
2 & Relative velocity between ego and center vehicles \\
3 & Front left vehicle position longitudinal \\
4 & Front left vehicle velocity \\
5 & Front left vehicle lateral position \\
6 & Front center vehicle position longitudinal \\
7 & Front center vehicle velocity \\
8 & Front center vehicle lateral position \\
9 & Front right vehicle position longitudinal \\
10 & Front right vehicle velocity \\
11 & Front right vehicle lateral position \\
12 & Rear left vehicle position longitudinal \\
13 & Rear left vehicle velocity \\
14 & Rear left vehicle lateral position \\
15 & Rear center vehicle position longitudinal \\
16 & Rear center vehicle velocity \\
17 & Rear center vehicle lateral position \\
18 & Rear right vehicle position longitudinal \\
19 & Rear right vehicle velocity \\
20 & Rear right vehicle lateral position \\
\hline
\end{tabular}

self-driving vehicle in diverse traffic conditions. More details can be found in [1].

The data set was divided into $80 \%$ for training and $20 \%$ for validation purposes as usual for such tasks [21]. We used 10-fold cross validation for the experimental setup. It is important to highlight that the analyzed dataset is imbalanced as illustrated in Fig. (5). However, due to the prototypebased nature of the hierarchical approach no pre-processing is required in this case.

\section{A. Performance Evaluation}

In order to evaluate the performance of the proposed method the accuracy index is considered. Accuracy is defined as follows:

$$
A C C(\%)=\frac{T P+T N}{T P+F P+T N+F N},
$$

where $T P, F P, T N, F N$ denote true and false, negative and positive respectively.

All the experiments were conducted with MATLAB 2018a using a personal computer with a $1.8 \mathrm{GHz}$ Intel Core i5 
processor, 8-GB RAM, and MacOS operating system. The experiments were executed using 10 -fold cross validation under the same ratio of training-to-testing sample sets. The following methods were used for comparison: K-nearest Neighbors (KNN) [22], Naive Bayes (NB) [23], Support Vector Machine (SVM) [24], Decision Tree [25], Random Forest [26], XGBoost [27], and Catboost [28]. Parameters for XGBoost and CatBoost were optmized through Auto-sklearn hyper-parameter optimization [29]. For the other state-of-theart approaches we consider the default parameters according to their references.

\section{Results And Analysis}

Computational simulations were performed to assess the accuracy of the explainable rule-based approach combined with the sensitivity selection method. Table II summarizes the results obtained by the proposed method considering different number of inputs.

Table II shows that, in general, the proposed autonomous method tends to be more accurate when the data space is reduced as we remove inputs with the lowest densities. Because of the parallel nature of the rule-based method, the sensitivity selection is able to work per action. Therefore, the proposed density-based input selection is able to create an individualized subset of inputs per action.

Table III, shows that, for the best scenario with 7 inputs (Table IV and Fig. (6) details the 7 inputs with higher densities for action 1, similar trend happened with all the actions). The proposed autonomous method reached the best result in terms of overall accuracy compared to the other state-of-theart methods. The rule-based method outperformed the other state-of-the-art approaches in terms of accuracy for 5 out of 8 actions. It is also possible to note through Table III that the SVM, Adaboost, Discriminant analysis, XGBoost, and CatBoost were not able to detect some of the actions, mainly actions 6 and 8 where the respective accuracy was $0 \%$. Actions 6 and 8, refer to rare maneuvers during the driving simulation, are extremely difficult to recognise due the highly imbalanced data set, as illustrated in Fig. (5).

When all available information is considered as model's inputs it may cause overfitting, and then be prejudicial to the model's accuracy. Therefore, when the proposed densitybased input selection method creates individualized subset of the most descriptive inputs per action it helps to overcome the dimensionality problem. For example, the overall accuracy of the proposed recommendation system increases from $94.54 \%$ to $98.94 \%$ using one third of the original dimensions of the input space, moreover, accuracy per action is also improved. Therefore, one can note that when a self-driving car needs to take an action such as "Lane change right and also brake by $-2 \mathrm{~m} / \mathrm{s}^{2}$ " a different subset of inputs will have more impact than when a self-driving car needs to "Lane change left and also brake by $-2 \mathrm{~m} / \mathrm{s}^{2 "}$ as different maneuvers require different set of actions by the driver/agent.

Fig. (7) illustrates the confusion matrix for the best scenario. It is notable from the confusion matrix that even though the data set is imbalanced, the proposed method is able to

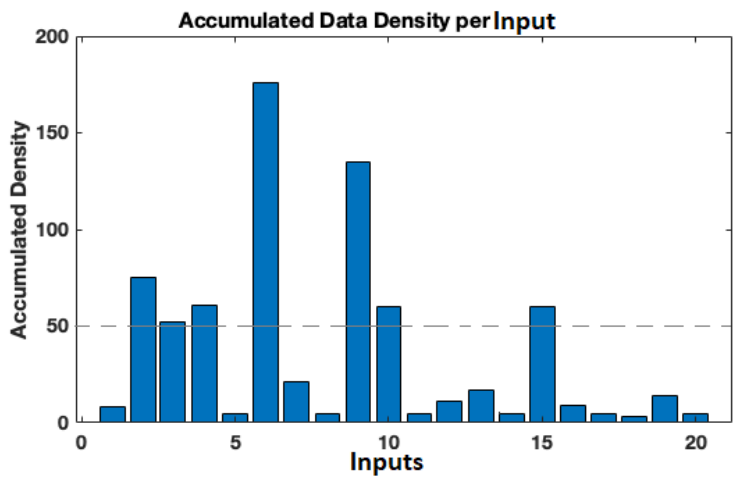

Fig. 6. Accumulated density histogram, density bars above the dotted line denote the top seven density inputs.

correctly identify uniformly all the actions. Fig. (8) illustrates how the performance is affected as the data space is reduced with the removal of inputs with the lowest densities. One can see from Fig. (8) that the sensitivity selection helps to improve the computational complexity by reducing the number of inputs (less calculations are required due to the $\mathrm{O}$ $(\log n)$ nature of the proposed approach).

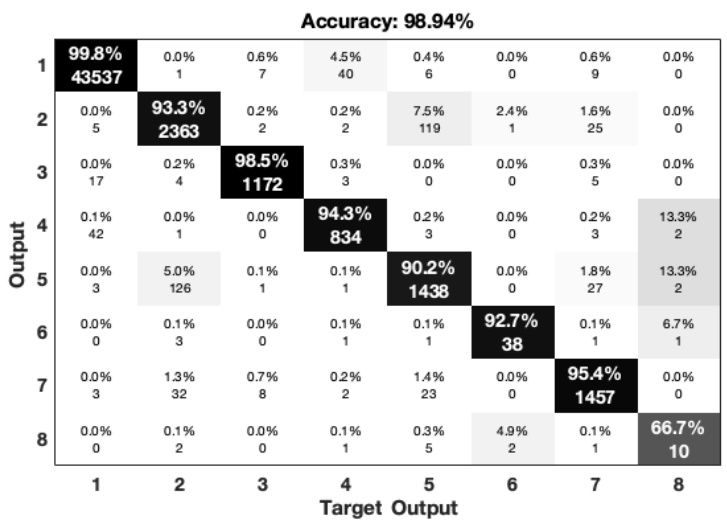

Fig. 7. Confusion matrix for the best scenario (7 inputs)

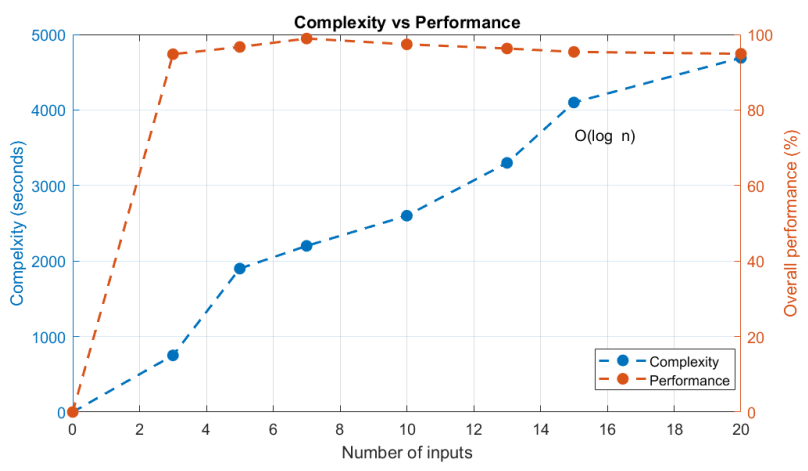

Fig. 8. Computational complexity vs Overall performance

Besides the improvement in terms of accuracy, the hierarchical method contributed to improving interpretability 
TABLE II

PERFORMANCE COMPARISON FOR DIFFERENT ACTIONS AND NUMBER OF INPUTS

\begin{tabular}{|c|c|c|c|c|c|c|c|c|c|}
\hline \# Inputs ${ }^{1} \quad$ Accuracy & Action 1 & Action 2 & Action 3 & Action 4 & Action 5 & Action 6 & Action 7 & Action 8 & Overall \\
\hline 20 & $98.46 \%$ & $85.03 \%$ & $75.52 \%$ & $49.46 \%$ & $80.16 \%$ & $66.66 \%$ & $92.96 \%$ & $50.0 \%$ & $94.54 \%$ \\
\hline 10 & $98.75 \%$ & $88.02 \%$ & $83.06 \%$ & $91.34 \%$ & $91.72 \%$ & $72.72 \%$ & $96.85 \%$ & $38.09 \%$ & $97.41 \%$ \\
\hline 7 & $\mathbf{9 9 . 8} \%$ & $\underline{\mathbf{9 3 . 3}} \%$ & $\mathbf{9 8 . 5} \%$ & $94.3 \%$ & $\overline{90.02} \%$ & $\mathbf{9 2 . 7} \%$ & $95.4 \%$ & $66.7 \%$ & 98.94\% \\
\hline 5 & $\overline{98.71} \%$ & $\overline{83.97 \%}$ & $\overline{81.66} \%$ & $\overline{78.06} \%$ & $87.91 \%$ & $\overline{80.00} \%$ & $\overline{95.84} \%$ & $76.47 \%$ & $\overline{96.75 \%}$ \\
\hline 3 & $98.36 \%$ & $84.43 \%$ & $77.92 \%$ & $39.92 \%$ & $78.98 \%$ & $81.08 \%$ & $93.06 \%$ & $66.67 \%$ & $94.89 \%$ \\
\hline
\end{tabular}

TABLE III

PERFORMANCE COMPARISON FOR DIFFERENT ACTIONS WITH 7 INPUTS WITH THE HIGHEST DENSITY

\begin{tabular}{|c|c|c|c|c|c|c|c|c|c|}
\hline Method Accuracy & Action 1 & Action 2 & Action 3 & Action 4 & Action 5 & Action 6 & Action 7 & Action 8 & Overall \\
\hline This paper & $\underline{99.8} \%$ & $\underline{\mathbf{9 3 . 3}} \%$ & 98.5\% & $94.3 \%$ & $90.02 \%$ & $92.7 \%$ & $95.4 \%$ & $66.7 \%$ & $9 \mathbf{9 8 . 9 4} \%$ \\
\hline This paper (MegaClouds) & $\overline{99.34} \%$ & $\overline{89.82} \%$ & $\overline{82.9} \%$ & $81.45 \%$ & $92.76 \%$ & $72.0 \%$ & $97.31 \%$ & $72.72 \%$ & $\overline{97.88} \%$ \\
\hline SVM & $88.65 \%$ & $53.6 \%$ & $0 \%$ & $0 \%$ & $100.0 \%$ & $\underline{100.0} \%$ & $74.7 \%$ & $0 \%$ & $87.08 \%$ \\
\hline KNN & $97.34 \%$ & $83.1 \%$ & $85.6 \%$ & $84.7 \%$ & $72.6 \%$ & $\underline{100.0} \%$ & $90.2 \%$ & $70.00 \%$ & $95.23 \%$ \\
\hline Decision Tree & $98.82 \%$ & $88.16 \%$ & $83.4 \%$ & $82.26 \%$ & $93.36 \%$ & $82.92 \%$ & $92.45 \%$ & $70.00 \%$ & $97.02 \%$ \\
\hline Adaboost & $94.3 \%$ & $72.9 \%$ & $88.0 \%$ & $97.9 \%$ & $87.9 \%$ & $0 \%$ & $86.0 \%$ & $0 \%$ & $92.95 \%$ \\
\hline Discriminant analysis & $91.0 \%$ & $42.3 \%$ & $33.9 \%$ & $0 \%$ & $36.6 \%$ & $32.8 \%$ & $38.6 \%$ & $10.3 \%$ & $85.43 \%$ \\
\hline Random Forest & $99.4 \%$ & $90.2 \%$ & $88.6 \%$ & $98.1 \%$ & $94.2 \%$ & $75.2 \%$ & $86.0 \%$ & $75.4 \%$ & $98.31 \%$ \\
\hline XGBoost & $99.2 \%$ & $84.5 \%$ & $86.9 \%$ & $\overline{87.3 \%}$ & $89.9 \%$ & $32.8 \%$ & $89.4 \%$ & $0 \%$ & $97.12 \%$ \\
\hline CatBoost & $93.2 \%$ & $78.7 \%$ & $90.1 \%$ & $92.2 \%$ & $88.2 \%$ & $52.3 \%$ & $87.1 \%$ & $0 \%$ & $91.45 \%$ \\
\hline
\end{tabular}

TABLE IV

DESCRIPTION OF THE 7 INPUTS WITH HIGHER DENSITIES

\begin{tabular}{c|c}
\hline Inputs & Description \\
\hline$R v$ & Relative velocity between Ego and center vehicles \\
$d^{F L}$ & Front left vehicle position longitudinal \\
$V^{F L}$ & Front left vehicle velocity \\
$d^{F C}$ & Front center vehicle position longitudinal \\
$d^{F R}$ & Front right vehicle position longitudinal \\
$V^{F R}$ & Front right vehicle velocity \\
$d^{B C}$ & Rear center vehicle position longitudinal \\
\hline
\end{tabular}

of the proposed approach. It allowed to infer a meaningful approximation of the DRL model and enabled quick evaluation of its performance for specific use cases. Table $\mathrm{V}$ details the number of prototypes produced by the proposed recommendation system considering different layers and 7 inputs (best scenario).

Generated trapezoidal fuzzy rules for the MegaClouds layer (highly abstract layer) can be illustrated in terms of inputs as illustrated by Fig. (9). It also can be visualized in terms of rules per prototype as given by Fig. (10).

Fig. (11) illustrates the actions given by the proposed method along the time. This is helpful to analyse the driving behavior and sequence of events by specialists.

In general, experiments have shown that the proposed explainable method is an efficient framework for this challenging task. Results showed advantages (98.94\%) compared to similar methods for addressing the approximation task. Moreover, the proposed method in its top layer also produced transparent linguistic fuzzy rules, which are human interpretable. In addition, the hierarchical architecture allows to reduce the rule antecedents and to simplify the structure of the rule-based models.

\section{CONClusion}

In this paper, we propose a novel explainable rule-based machine learning model that can be used to approximate the decisions policy of a DRL agent. To generate training data we used a DRL model representing a highway path planning policy for autonomous driving. The model is composed of a 0-order fuzzy rules. Experiments have shown that the proposed method was able to produce more accurate results than the other similar state-of-the-art methods.

We also present a new hierarchical mechanism to significantly reduce the number of generated fuzzy rules. In this case, adjacent (in the data space) prototypes which correspond to the same action are grouped and merged into so-called "MegaClouds". The proposed method helped to improve the interpretability of the generated models. Moreover, the input selection method based on ranking the density per input dimension in the data space contributed to improve the accuracy of the models as it creates individualized subsets of inputs per action, taking advantage of the parallel characteristic of the proposed explainable self-organizing method. Experimental results show that an accurate and computationally efficient explainable alternative to the deep neural network model can be successfully developed providing opportunities to explain and validate the decisions by the DRL agent.

\footnotetext{
${ }^{1}$ Inputs with the highest density
} 
TABLE V

NUMBER OF IDENTIFIED PROTOTYPES PER ACTION FOR DIFFERENT HIERARCHICAL LAYERS

\begin{tabular}{c|ccccccccc}
\hline \# Prototypes & Action 1 & Action 2 & Action 3 & Action 4 & Action 5 & Action 6 & Action 7 & Action 8 & Total \\
\hline Bottom Layer & 1315 & 1009 & 482 & 360 & 649 & 17 & 607 & 4 & 4443 \\
MegaClouds & 13 & 14 & 8 & 10 & 15 & 6 & 11 & 4 & $\underline{\mathbf{8 1}}$ \\
\hline
\end{tabular}

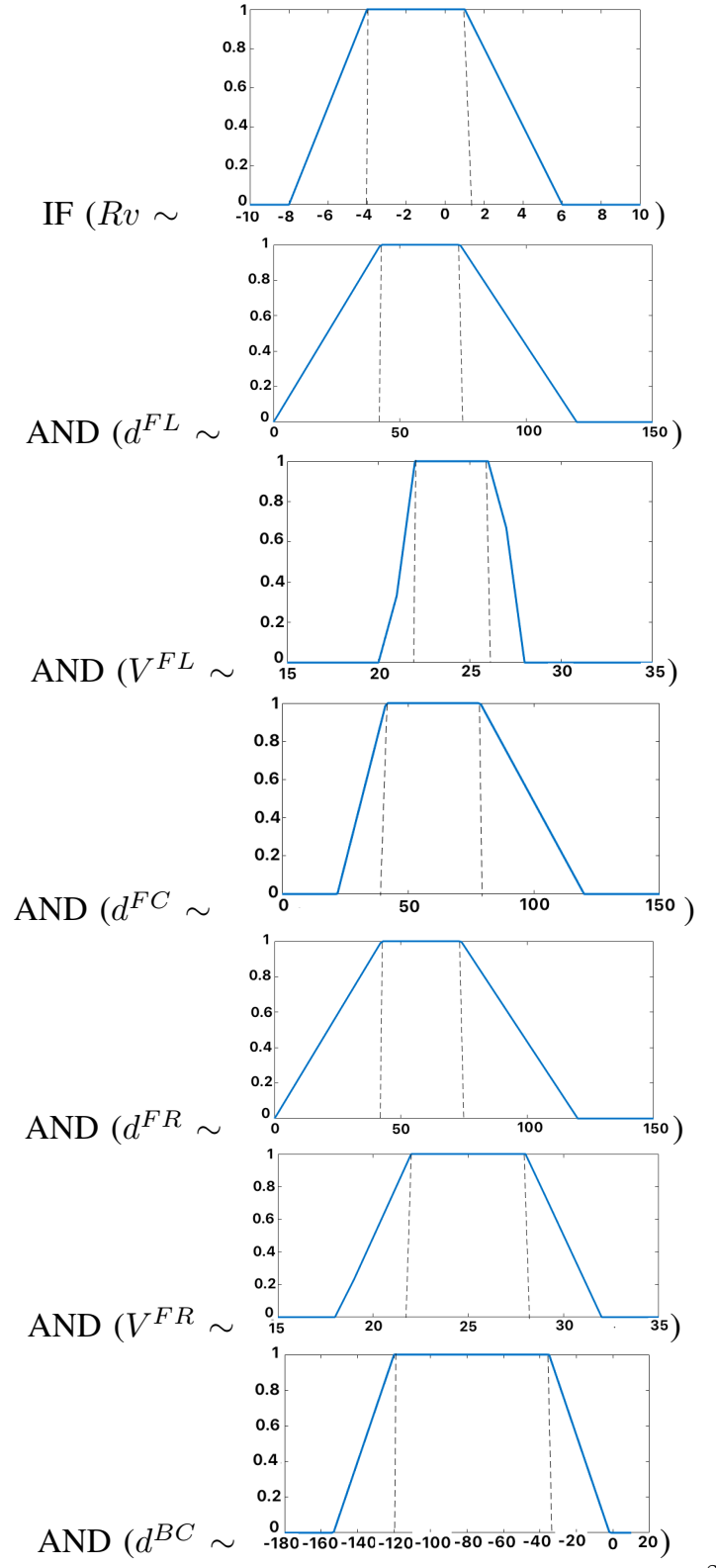

THEN 'Lane change left and also brake by $-2 \mathrm{~m} / \mathrm{s}^{2}$,

Fig. 9. Trapezoidal rule per feature for 'Lane change left and also brake by $-2 \mathrm{~m} / \mathrm{s}^{2}$, (Action 6)

\section{ACKNOWLEDGMENT}

The first two authors would like to thank Ford Motor Company for the financial and technical support. This work is part of the URP project "Transparent Deep Learning Classifier of Driving Scenarios able to Identify and Learn

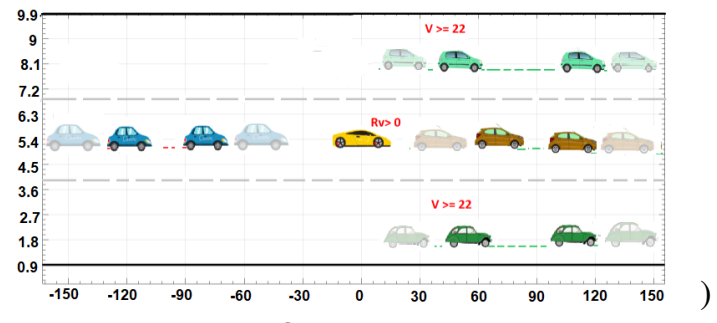

IF $(x \sim$

OR

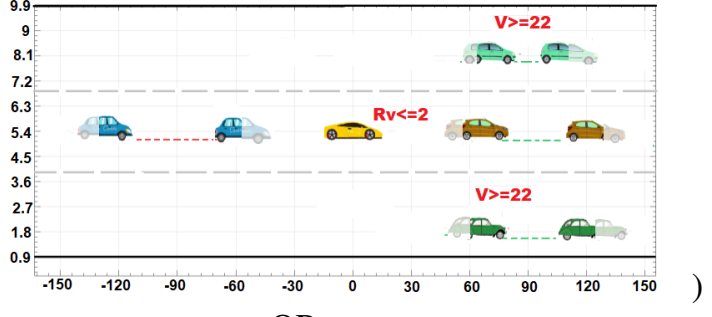

OR

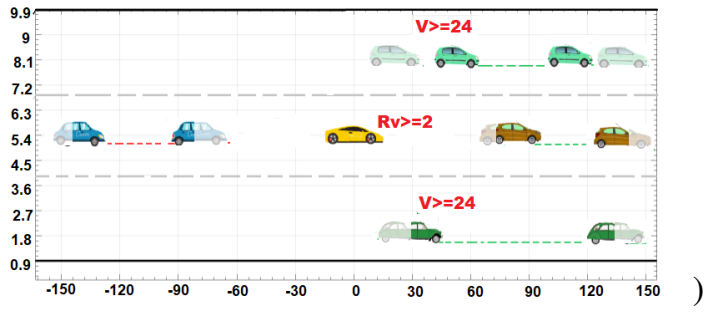

OR ... OR

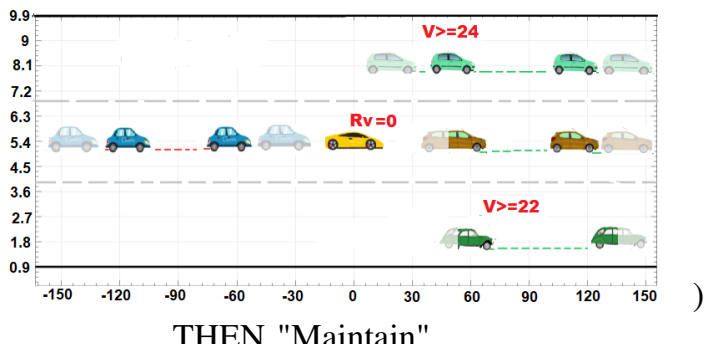

Fig. 10. Visual interpretation of trapezoidal rule for "Maintain" (Action 1), where the watermarked cars represent the soft trapezoidal fuzzy boundaries and the solid cars denotes the limits of the MegaClouds. $R_{v}$ denotes the relative velocity between $\mathrm{EV}$ and center vehicle, and $V$ denotes the velocities for the front left and front right vehicles, both $R_{v}$ and $V$ are in $\mathrm{m} / \mathrm{s}$

from Unseen Situations".

\section{REFERENCES}

[1] S. Nageshrao, H. E. Tseng, and D. Filev, "Autonomous highway driving using deep reinforcement learning," in 2019 IEEE International Conference on Systems, Man and Cybernetics (SMC). IEEE, 2019, pp. 2326-2331.

[2] H. Van Hasselt, A. Guez, and D. Silver, "Deep reinforcement learning with double q-learning," in Thirtieth AAAI conference on artificial intelligence, 2016. 


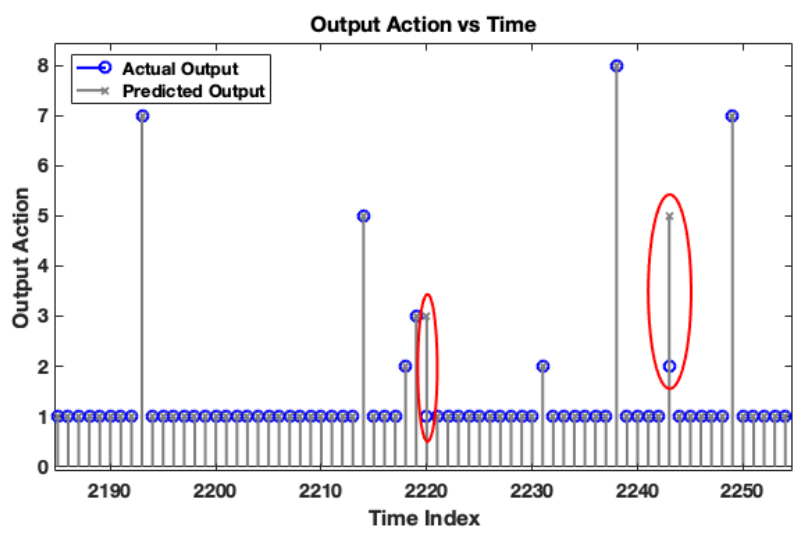

Fig. 11. Action vs time. The red ellipsoid indicates the wrongly predicted action given by the proposed approach

[3] M. Coeckelbergh, "Artificial intelligence, responsibility attribution, and a relational justification of explainability," Science and engineering ethics, pp. 1-18, 2019.

[4] G. Wiegand, M. Schmidmaier, T. Weber, Y. Liu, and H. Hussmann, "I drive-you trust: Explaining driving behavior of autonomous cars," in Extended Abstracts of the 2019 CHI Conference on Human Factors in Computing Systems, 2019, pp. 1-6.

[5] S. Ramos, S. Gehrig, P. Pinggera, U. Franke, and C. Rother, "Detecting unexpected obstacles for self-driving cars: Fusing deep learning and geometric modeling," in Intelligent Vehicles Symposium (IV), 2017 IEEE. IEEE, 2017, pp. 1025-1032.

[6] J. Stilgoe, "Machine learning, social learning and the governance of self-driving cars," Social studies of science, vol. 48, no. 1, pp. 25-56, 2018.

[7] I. Škrjanc, G. Andonovski, A. Ledezma, O. Sipele, J. A. Iglesias, and A. Sanchis, "Evolving cloud-based system for the recognition of drivers' actions," Expert Systems with Applications, vol. 99, pp. 231238, 2018.

[8] G. Gutierrez, J. A. Iglesias, F. J. Ordoñez, A. Ledezma, and A. Sanchis, "Agent-based framework for advanced driver assistance systems in urban environments," in 17th International Conference on Information Fusion (FUSION). IEEE, 2014, pp. 1-8.

[9] P. P. Angelov and X. Gu, Empirical approach to machine learning. Springer, 2018, ISBN: 978-3-030-02384-3.

[10] J. A. Iglesias, P. Angelov, A. Ledezma, and A. Sanchis, "Evolving classification of agents' behaviors: a general approach," Evolving Systems, vol. 1, no. 3, pp. 161-171, 2010.

[11] C. Rudin, "Stop explaining black box machine learning models for high stakes decisions and use interpretable models instead," Nature Machine Intelligence, vol. 1, no. 5, pp. 206-215, 2019.

[12] G. Andonovski, G. Mušič, S. Blažič, and I. Škrjanc, "Evolving model identification for process monitoring and prediction of non-linear systems," Engineering applications of artificial intelligence, vol. 68, pp. 214-221, 2018.

[13] P. P. Angelov and D. P. Filev, "An approach to online identification of takagi-sugeno fuzzy models," IEEE Transactions on Systems, Man, and Cybernetics, Part B (Cybernetics), vol. 34, no. 1, pp. 484-498, 2004.

[14] P. Angelov and X. Gu, "Autonomous learning multi-model classifier of 0-order (almmo-0)," in 2017 Evolving and Adaptive Intelligent Systems (EAIS). IEEE, 2017, pp. 1-7.

[15] P. P. Angelov and X. Gu, "Deep rule-based classifier with human-level performance and characteristics," Information Sciences, vol. 463-464, pp. 196-213, 2018.

[16] T. Hastie, R. Tibshirani, and J. Friedman, The elements of statistical learning: data mining, inference, and prediction. Springer Science \& Business Media, 2009.

[17] P. Angelov, Autonomous learning systems: from data streams to knowledge in real-time. John Wiley \& Sons, 2012.

[18] Q. Du, V. Faber, and M. Gunzburger, "Centroidal voronoi tessellations: Applications and algorithms," SIAM review, vol. 41, no. 4, pp. 637676, 1999.
[19] H. Lee-Kwang, Y.-S. Song, and K.-M. Lee, "Similarity measure between fuzzy sets and between elements," Fuzzy Sets and Systems, vol. 62, no. 3, pp. 291-293, 1994.

[20] E. Soares, P. Angelov, D. Filev, B. Costa, M. Castro, and S. Nageshrao, "Explainable density-based approach for self-driving actions classification," in 2019 18th IEEE International Conference On Machine Learning And Applications (ICMLA). IEEE, 2019, pp. 469-474.

[21] K. K. Dobbin and R. M. Simon, "Optimally splitting cases for training and testing high dimensional classifiers," BMC medical genomics, vol. 4, no. 1, p. 31, 2011.

[22] P. Cunningham and S. J. Delany, "k-nearest neighbour classifiers," Multiple Classifier Systems, vol. 34, no. 8, pp. 1-17, 2007.

[23] I. Rish et al., "An empirical study of the naive bayes classifier," in IJCAI 2001 workshop on empirical methods in artificial intelligence, vol. 3, no. 22. IBM New York, 2001, pp. 41-46.

[24] J. A. Suykens and J. Vandewalle, "Least squares support vector machine classifiers," Neural processing letters, vol. 9, no. 3, pp. 293300, 1999.

[25] S. R. Safavian and D. Landgrebe, "A survey of decision tree classifier methodology," IEEE transactions on systems, man, and cybernetics, vol. 21, no. 3, pp. 660-674, 1991.

[26] L. Breiman, "Random forests," Machine learning, vol. 45, no. 1, pp 5-32, 2001.

[27] T. Chen and C. Guestrin, "Xgboost: A scalable tree boosting system," in Proceedings of the 22nd acm sigkdd international conference on knowledge discovery and data mining, 2016, pp. 785-794.

[28] L. Prokhorenkova, G. Gusev, A. Vorobev, A. V. Dorogush, and A. Gulin, "Catboost: unbiased boosting with categorical features," in Advances in neural information processing systems, 2018, pp. 66386648.

[29] M. Feurer, A. Klein, K. Eggensperger, J. Springenberg, M. Blum, and F. Hutter, "Efficient and robust automated machine learning," in Advances in neural information processing systems, 2015, pp. 29622970. 